\title{
Test Methods and Results for Sensors in a Pre-Crash Detection System
}

\author{
Charles Birdsong, Ph.D., Peter Schuster, Ph.D., John Carlin, Daniel Kawano, William \\ Thompson \\ California Polytechnic State University, San Luis Obispo, California
}

Copyright @ 2005 SAE International

\begin{abstract}
Automobile safety can be improved by anticipating a crash before it occurs and thereby providing additional time to deploy safety technologies. This requires an accurate, fast and robust pre-crash sensor that measures telemetry, discriminates between classes of objects over a range of conditions, and has sufficient range and area of coverage surrounding the vehicle. The sensor must be combined with an algorithm that integrates data to identify threat levels. No one sensor provides adequate information to meet these diverse and demanding requirements. However the requirements can be met with an optimal combination of multiple types of sensors. Previous work considered criteria for evaluating various sensors to find an optimal combination. This work presents test methods and results for selected sensors proposed for use in a precrash detection system. The test methods include static and dynamic telemetry testing to identify the range, accuracy, reliability and operating conditions for each sensor. Each sensor is evaluated for its ability to discriminate between classes of objects. The tests are applied to ultrasonic, laser range finder and radar sensors. These sensors were selected because they provide the maximum information, cover a broad range and region and are commercially viable in passenger vehicles.
\end{abstract}

\section{INTRODUCTION}

Motor vehicle crashes are the leading cause of death for persons of every age from 2 through 33 . Since the 1960s, introduction of passive safety equipment (e.g. seat belts, air bags, crush structures) has dramatically reduced accident rates, injury severity and the number of fatalities, however the absolute number of deaths and injuries remains high. Since 1993, every year nearly 6 million motor vehicle crashes have consistently resulted in over 40,000 deaths in the US alone (NHTSA 2005). Certain conditions (weather, lighting, impairment, distraction) limit drivers' effectiveness at recognizing and responding to dangerous situations. For example, 50\% of fatal accidents occur outside of daylight hours, and $12 \%$ during inclement weather. Driver distraction is cited as a contributing cause in half of all accidents.

In order to significantly reduce accident severity and occurrence, future safety technologies must move beyond 'passive.' To support this, vehicles will require new exterior pre-crash sensors to create an electronic awareness of the traffic situation. Pre-crash sensing may well have the most impact in reducing injuries from nighttime accidents involving impaired drivers. However, the advanced safety features enabled by precrash sensing will provide a significant benefit in all cases of poor lighting, bad weather, or driver distraction.

Figure 1 illustrates some near-term safety benefits of pre-crash sensing. Current vehicles (top half of the figure) do not have any means of anticipating a crash. In the short time frame (approximately 10-20 ms) after a crash is detected by acceleration-based sensors the options for deploying safety technologies is limited. Currently airbags are deployed approximately $10-20 \mathrm{~ms}$ after impact and must be inflated rapidly so that they are in place to protect the passenger. If the crash could be anticipated then additional time would be available to deploy new safety technologies such as audible alarms, seatbelt pre-tensioners, automatic door locks, seat stiffeners, seat position control, window closing, slower airbag inflation rates, and pre-crash braking (Lyons \& Taskin 2000, Spies 2002, Knoll et al. 2004). The result would be increased vehicle crash survival rates. In addition, pre-crash detection will reduce the incidence of unnecessary airbag deployment. Studies show that unnecessary airbag deployment can cause greater injuries than a minor crash would cause (Jones 2002). 


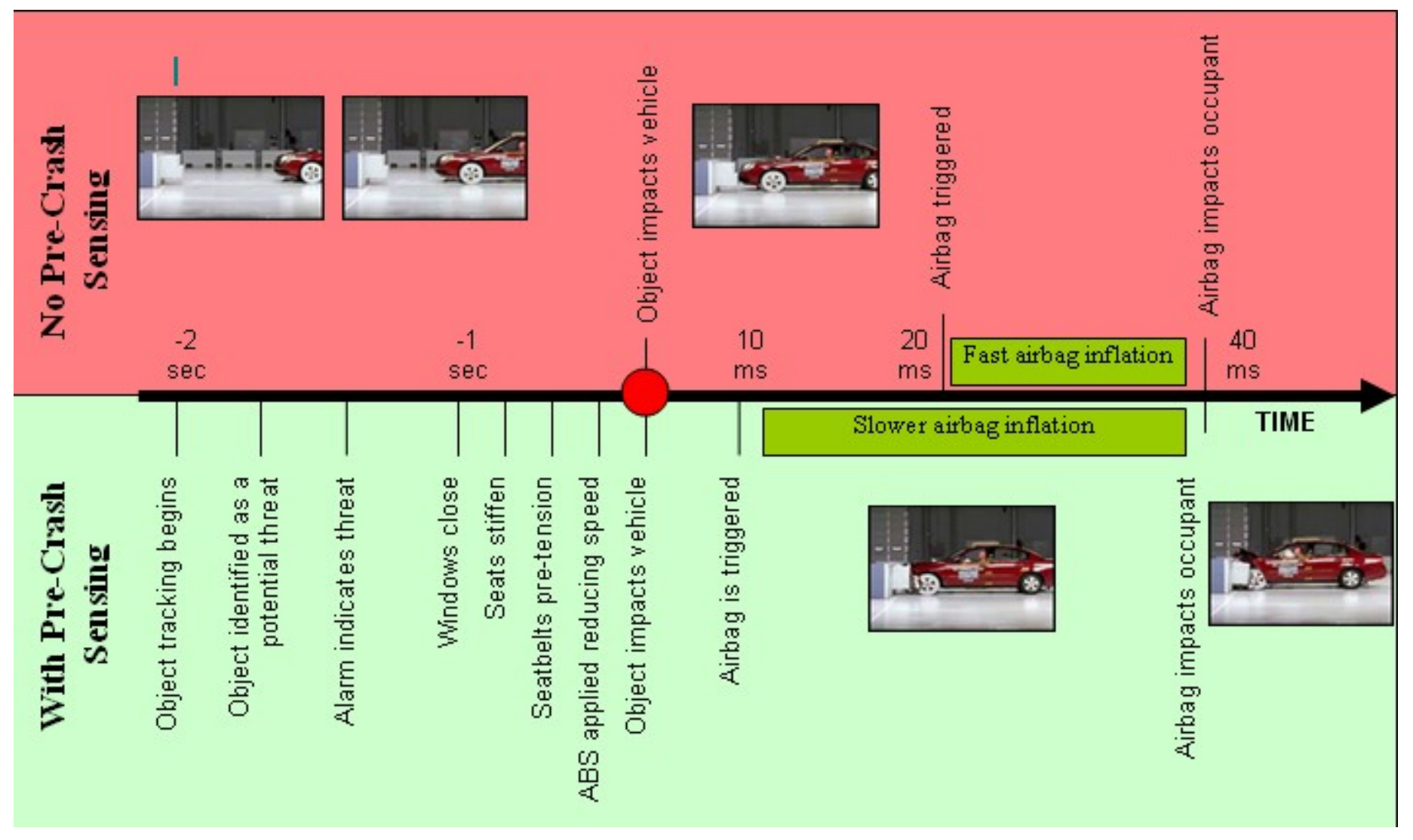

Figure 1: Timelines for collisions with and without pre-crash sensing

Beyond the passive safety technologies shown in Figure 1 , an advanced pre-crash sensing system will also be capable of directing future accident-avoidance technologies. For example, an automated braking system could augment a driver's braking force if the sensor determines more deceleration is necessary to stop the vehicle before impact. With increased sensor robustness, this system could be used to automatically apply the brakes when an imminent crash is predicted; regardless of whether braking is already applied.

This type of technology is not new. Certain external sensors have already been adopted into passenger vehicles, reducing the costs and broadening consumer acceptance. For example, ultrasonic sensors are used as parking aids on many vehicles, passive infrared sensors have been used to aid vision at night, and radars are used in adaptive cruise control (ACC) systems to maintain safe following distance when cruise control is active. These sensors are used in a passive sense and not to actively avoid or aid in a crash event. However it has been proposed that they could be integrated with intelligent real-time algorithms to do so (Knoll et al. 2004). This requires an accurate, fast and robust pre-crash sensor that measures telemetry, discriminates between classes of objects over a range of conditions, and has sufficient range and area of coverage surrounding the vehicle. The sensor must be combined with an algorithm that integrates data to identify threat levels. No one sensor provides adequate information to meet these diverse and demanding requirements. However the requirements can be met with an optimal combination of multiple types of sensors. Previous work considered criteria for evaluating various sensors to find an optimal combination (Carlin, et. al. 2005).

To support the development of future integrated precrash sensing systems, this paper presents methods for testing individual sensors in a pre-crash detection system. In most cases, the proposed sensors were not developed for pre-crash detection, but may be adapted to this use. As a result, sensor performance for this application is not already known. Pre-crash detection is a new function that is distinct from other types of sensor applications so new test protocols are required.

The objective of a pre-crash sensor is to provide telemetry and object discrimination data at a suitable range and rate to predict a crash event. Accuracy, reliability and environmental factors must be considered as well. Test methods can be collected into general categories of static, dynamic, and object discrimination with specific procedures defined in each category. In addition to the test methods, preliminary test results are presented in this paper. These are intended as examples that can be used for a broad range of proposed pre-crash sensors. 


\section{STATIC TESTS}

Static tests measure the performance of sensors while both targets and sensors are stationary. This is expected to provide a measure of the optimal sensor performance since there is no relative motion. Static tests include range, accuracy, and reliability. Each of these criteria is evaluated for sensitivity to multiple objects, vibrations, and environmental factors.

\section{RANGE, ACCURACY, AND RELIABILITY}

Distance Range measures the minimum and maximum distances at which the sensor can detect objects with reasonable accuracy. The sensor manufacturer usually provides this information for certain standard objects, with a factor of safety to account for environmental conditions and other factors. Under ideal cases a sensor's range may exceed the manufacturer's specification significantly. The test method involves placing a target a fixed distance from the sensor and comparing the sensor output with the distance measured using a tape measure. The range can depend on the shape, surface finish or material of the target. For example a LIDAR may have a longer range for reflective surfaces compared with a dispersive surface. An ultrasonic sensor may have longer range with large flat surfaces (walls) versus smaller curved surfaces (pole, ball, human). Minimum range information is also important because the most critical measurement for a pre-crash sensor triggering irreversible countermeasures is distinguishing a near miss from an actual crash event. Long-range data is less sensitive because far objects represent less of a threat (and proposed long-range countermeasures are currently reversible).

Field of View (FOV) Range measures the angular detection range of the sensor. LIDAR measures in a straight line (narrow beam), but RADAR and ultrasonic sensors have a cone of coverage. A signal is broadcast and reflected by the target and the reflection is used to obtain the telemetry data. The FOV is important in designing a pre-crash sensor to avoid blind spots near the sensor where critical measurement is needed. Also, if multiple sensors are used, the FOV can be used to develop an algorithm to more effectively integrate output signals. The FOV test method involves placing a target at specific positions at pre-identified distance and angle from the centerline of the sensor. The sensor output is compared to a target distance measured with a tape measure and protractor from the center of the sensor. The results can be reduced into a map that shows the area of coverage in detail. Typically the data from the periphery of the angular range is unreliable and the FOV is defined by the locations where the data is accurate and repeatable. Figure 2 provides an example of the output map obtained from testing an Ultrasonic sensor.

Accuracy indicates how well the sensor predicts the telemetry data compared to a known value. The data in Figure 3 shows range test results with an ultrasonic sensor for various objects. The graph records the absolute deviation of the measured distance of the target predicted by the sensor compared to the measured distance with a tape measure. The results indicate that the ultrasonic sensor has accurate range from about 0.1 meters up to 4 meters with little sensitivity to different target objects. Beyond 4 meters some objects can be measured while others cannot. The difference is likely due to the ability of the target surface and shape to reflect the ultrasonic waves back to the sensor.

Reliability is defined to be the probability that the sensor will not fail (i.e. return erroneous results) in any given measurement. A safety sensor is expected to have extremely high reliability. However, for the purposes of a new safety technology that is intended to enhance (significantly) the already-proven performance of passive safety systems, the important aspect of reliability is that the system should do no harm. In other words, while the long-term goal is a system that can detect virtually all objects in virtually all conditions, a short-term aim is to detect as many as possible, with NO false detections. False positive detections are undesired because they might be used to trigger irreversible deployments or potentially drastic avoidance maneuvers. So, in the initial system requirements, avoiding false positives is more important than detecting every real contact.

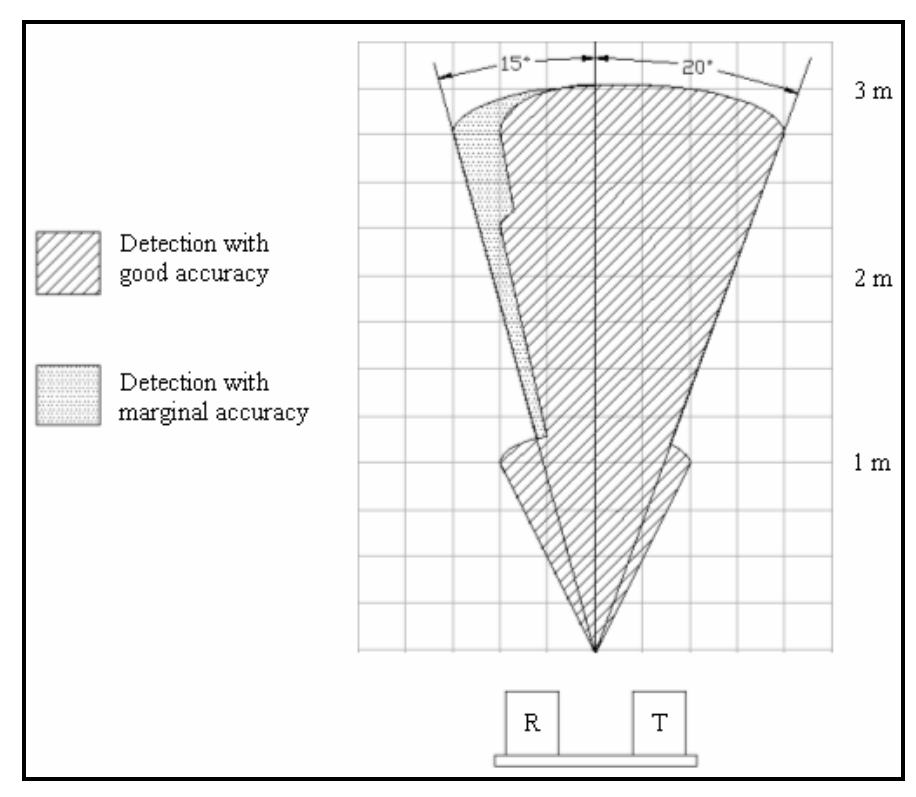

Figure 2: Ultrasonic rangefinder sonic cone map 


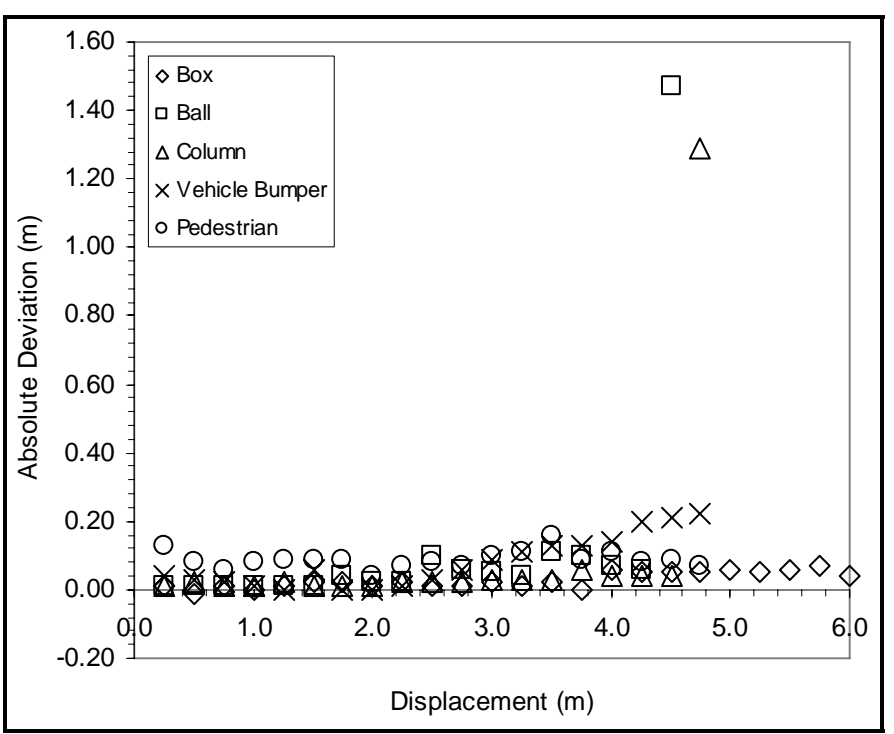

Figure 3: Ultrasonic rangefinder absolute deviation as a function of displacement for various target objects in static tests

The static test results for a LIDAR are presented in the figures below. Figure 4 shows that the accuracy of the LIDAR is well within in the $\pm 0.30 \mathrm{~m}$ claim of the manufacturer. In fact, on average all of the data falls within $\pm 0.20 \mathrm{~m}$ of the actual object distances. In general, it appears that the LIDAR tends to slightly overestimate the distance for most objects while underestimating for a pedestrian.

The absolute deviation remains within $\pm 0.20 \mathrm{~m}$ as distance is increased, and as a result, the percent error in the distance measurement decreases as distance is increased (Figure 5). When ranges are less than $5 \mathrm{~m}$, the $\pm 0.20 \mathrm{~m}$ accuracy causes significant errors. Beyond $5 \mathrm{~m}$, the effect of this accuracy is much less important.

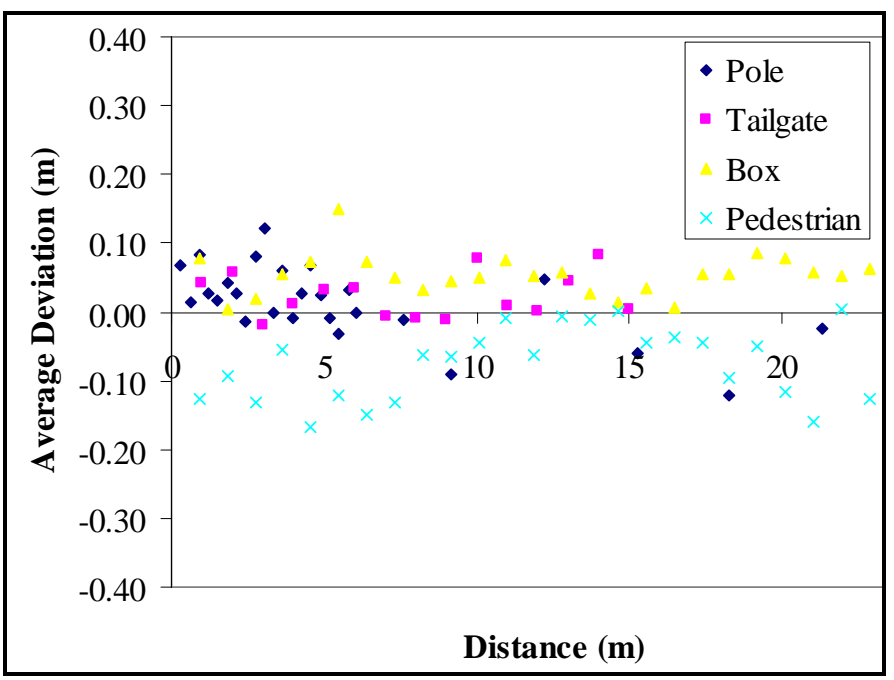

Figure 4: LIDAR absolute deviation as a function of distance for various objects in static testing

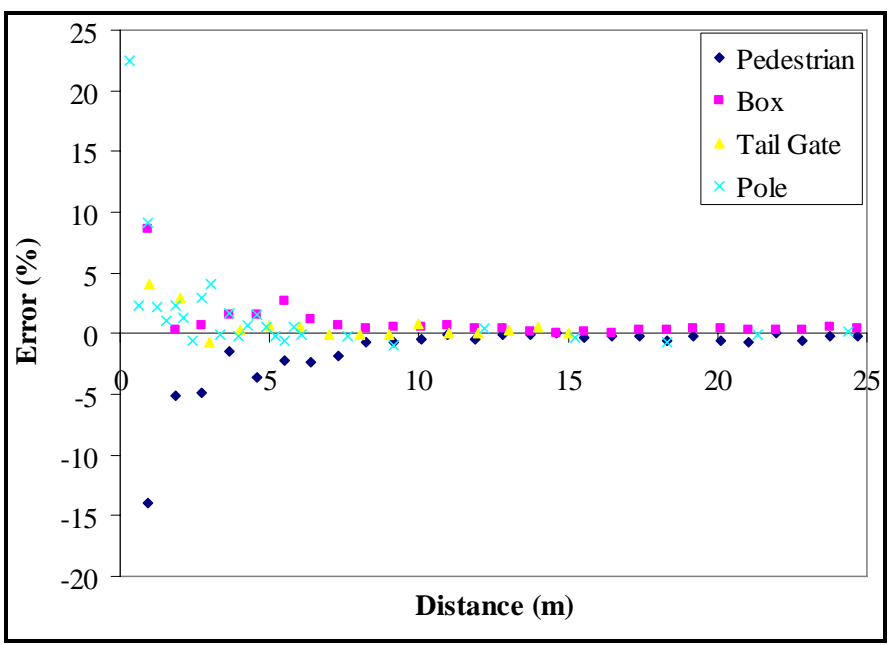

Figure 5: LIDAR percent error in distance measurement as a function of distance for various objects in static tests

\section{SENSITIVITY OF RESULTS}

Multiple Objects - These tests are intended to determine the sensor's ability to differentiate between the target (typically the object presenting the greatest threat to the vehicle) and other objects within range. In the real-world scenario, multiple objects will always be present, and a sensor must have some way of either (a) tracking multiple objects (e.g. most radar systems), or (b) selecting and reporting data on the most important objects (e.g. ultrasonic sensors give distance to only the closest object). Multiple object testing is currently underway

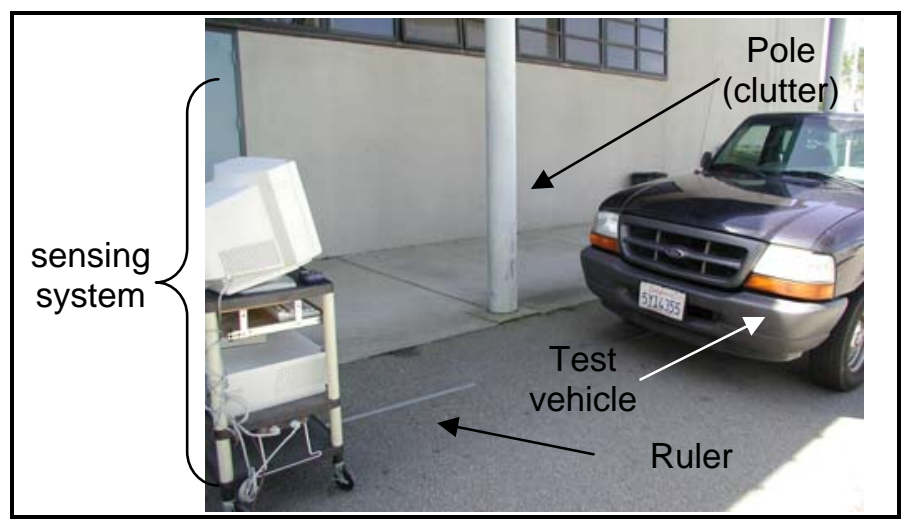

Figure 6: Experimental setup for multiple object static testing used in all sensor types

Environmental Factors - The environmental issues faced by an external automotive sensor are well understood. Many sensors proposed for a pre-crash system will have already been subjected to these conditions to evaluate their durability. However, evaluating the performance of the sensors under adverse conditions, and particularly under conditions known to cause issues for the tested sensor, are key to understanding the best way to integrate a number of sensors into a single system. 
Vibration - Vibration inputs into the exterior sensors depend to a certain extent on the specific vehicle and locations chosen for a particular application. However, as with environmental factors, typical automotive vibration loads are well understood and many sensors will already have been evaluated for durability. But, the performance of the sensors under these predicted vibrations is critical for evaluating their input to the integrated system.

\section{DYNAMIC TESTS}

Dynamic tests measure the performance of the sensors as the target or sensor moves during the test. Under some conditions the sensor's performance is degraded when there is relative motion. Dynamic tests are performed with controlled, usually fixed, velocities of the target object. Actual distance is determined using a separate, direct measurement scheme. In addition to determining the accuracy of a sensor under these circumstances, the dynamic tests enable additional assessment of sensitivity to direction of motion, near misses, detection time, and multiple objects.

Figure 7 shows the test setup for a simple $1 \mathrm{~m} / \mathrm{s}$ test of the Ultrasonic system using a string pot, while Figure 8 and Figure 9 give typical results. As the figures show, overall the sensor accurately measures the displacement of the test object (with occasional scatter), with the exception of the pole (95 $\mathrm{mm}$ diameter) as a target. Multiple trial runs with the pole indicated that the sensor consistently underestimates the displacement of the pole.

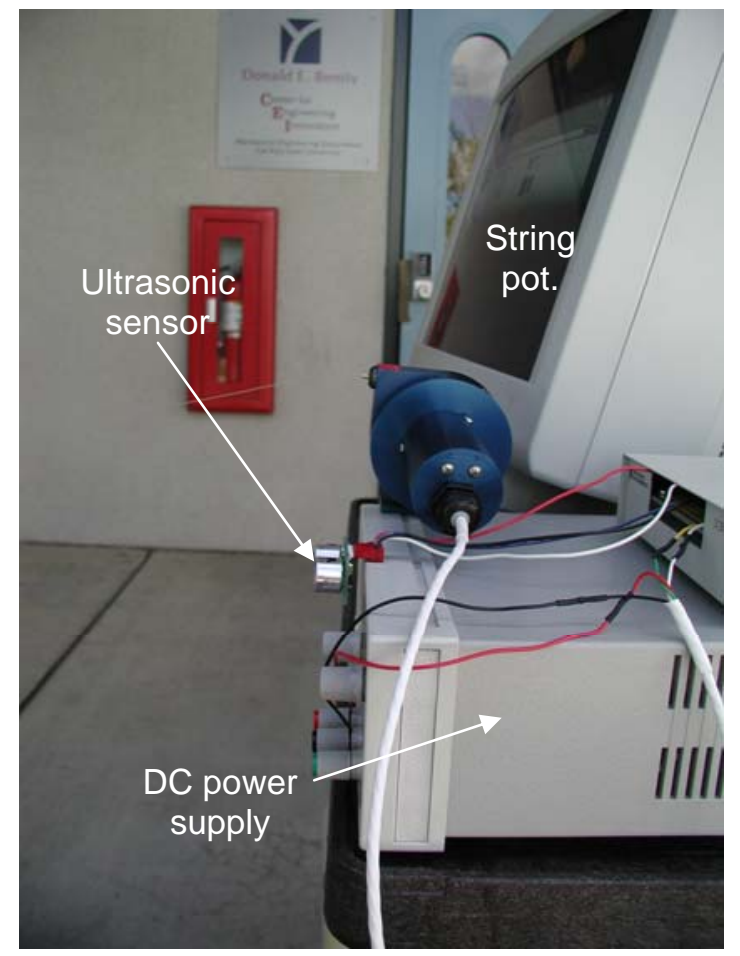

Figure 7: Ultrasonic sensor dynamic tests with string pot.

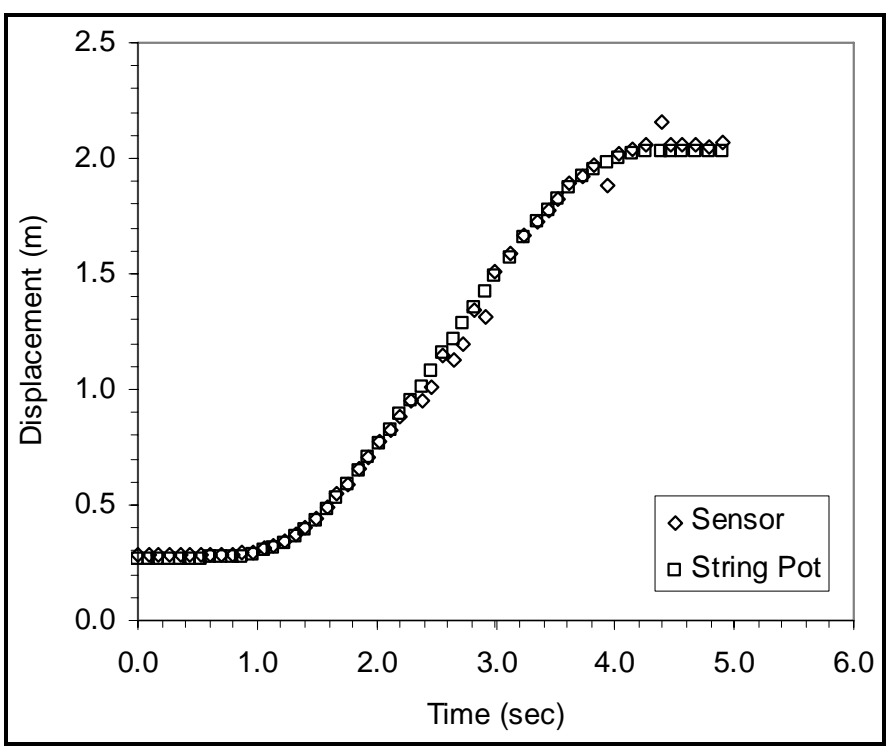

Figure 8: Typical results for dynamic test of ultrasonic sensor compared with string pot.

Time to Detection - A related performance criteria evaluated through dynamic testing is the time it takes a sensor to detect an object when it enters the FOV. Two conditions will be considered - motion laterally into the FOV from outside the angular range, and motion longitudinally into the FOV from outside the distance range.

Near Miss - For a system that triggers irreversible deployments or avoidance maneuvers, the sensors must be capable of predicting when a collision becomes unavoidable (i.e., no maneuvering can prevent it from occurring). The key criterion here is the difference between an impact and a near miss. For a head-on collision with significant offset, this may not be apparent until a few meters before contact. This criterion will be measured by assessing how the sensor output data changes for longitudinally moving objects located progressively further from the sensor centerline. 


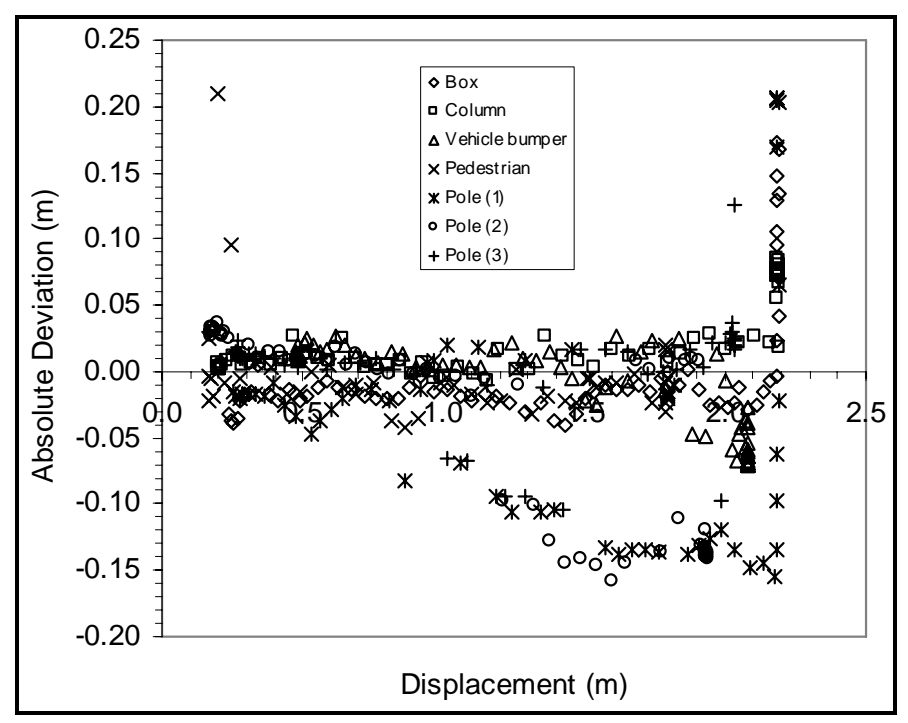

Figure 9: Absolute deviation as a function of displacement for various test objects used in the dynamic testing

Sensitivity to Direction - Many of the dynamic tests will be performed with object motion either directed longitudinal (parallel to the centerline of the sensor FOV), or transverse (perpendicular to the centerline of the sensor FOV). However, additional sensor tests are required to evaluate objects traveling within the FOV on trajectories between these extremes. Tests will be performed for motion vectors every $30^{\circ}$ between $0^{\circ}$ and $90^{\circ}$.

Multiple Objects - The goal of the dynamic multiple object tests is to determine how well a system responds when objects appear to join with and separate from each other in the FOV, or when the primary threat target changes from one object to another as relative positions change. The tests performed will include both lateral and longitudinal movement of two or more objects within the field of view.

\section{OBJECT DISCRIMINATION}

Object discrimination tests assess an additional aim of pre-crash sensing systems - the ability to distinguish between types of objects. The goal of these tests is to identify whether a particular sensor exhibits any differences in output signals with different objects (all other conditions held constant). The categories of objects for which different vehicle responses may be desired are summarized in Table 1. Sensor responses to each of these types of objects will be measured initially in static tests.
Table 1: Object types for discrimination tasks

\begin{tabular}{|l|l|l|l|l|}
\hline $\begin{array}{l}\text { Type of } \\
\text { object }\end{array}$ & Size & Mass & Type & $\begin{array}{l}\text { Example } \\
\text { object }\end{array}$ \\
\hline $\begin{array}{l}\text { Wide high } \\
\text { mass }\end{array}$ & Wide & High & Hard & $\begin{array}{l}\text { Tree, vehicle, } \\
\text { walls }\end{array}$ \\
\hline $\begin{array}{l}\text { Narrow } \\
\text { high mass }\end{array}$ & $\begin{array}{l}\text { Narro } \\
\text { w }\end{array}$ & High & Hard & Tree, pole \\
\hline $\begin{array}{l}\text { Wide low } \\
\text { mass }\end{array}$ & Wide & Med & Med & $\begin{array}{l}\text { Brush, } \\
\text { billboard, } \\
\text { motorcycle }\end{array}$ \\
\hline $\begin{array}{l}\text { Medium } \\
\text { high mass }\end{array}$ & Med & Med & Soft & $\begin{array}{l}\text { cow, moose, } \\
\text { pedestrian, } \\
\text { cyclist }\end{array}$ \\
\hline $\begin{array}{l}\text { Medium } \\
\text { low mass }\end{array}$ & $\begin{array}{l}\text { Narro } \\
\text { w }\end{array}$ & Med & Hard & Signpost \\
\hline Med & High & Hard & Boulder, Barrier \\
\hline $\begin{array}{l}\text { Small high } \\
\text { mass }\end{array}$ & $\begin{array}{l}\text { Narro } \\
\text { w }\end{array}$ & Low & Soft & $\begin{array}{l}\text { Small animals, } \\
\text { cones }\end{array}$ \\
\hline
\end{tabular}

The first set of testing evaluated the LIDAR reported signal strength. Since signal strength is related to the surface color and texture of the target, it may be useful for object discrimination. However, initial test results shown in Figure 10 indicate a weak correlation between object type and signal strength.

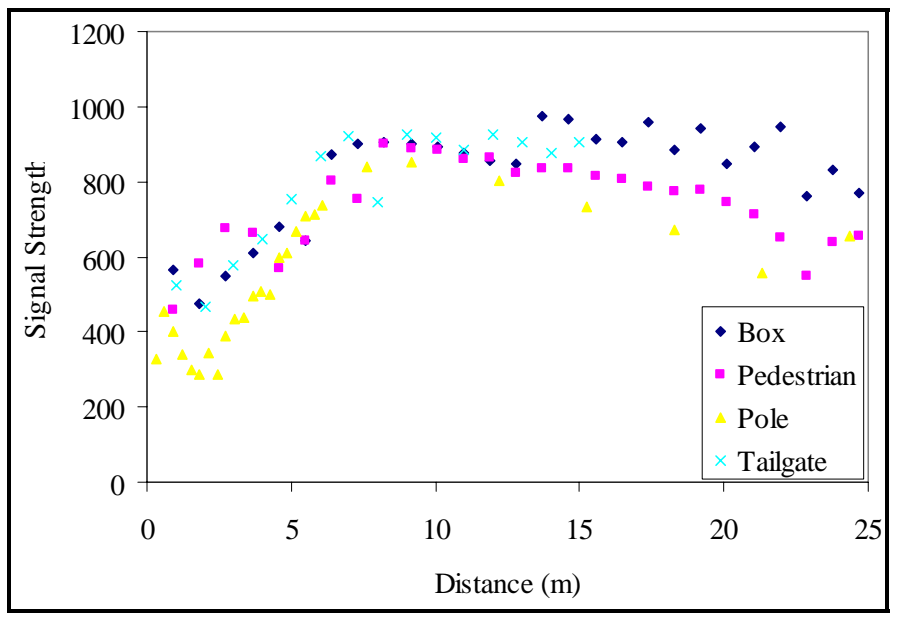

Figure 10: LIDAR signal strength as a function of distance for various objects in static tests

These results also indicate one major limitation of the LIDAR: it does not detect certain objects. For example, in the static test for the Ultrasonic sensor, the flat black bumper of a pickup truck was used rather than the tailgate. Upon targeting the bumper, the LIDAR returned a signal strength of zero. Further investigation has found that the range finder is not good at sensing black objects in general. The sensor could not detect a black trash bag or the black plastic back of a chair; however, it could detect the black nylon of a backpack. More testing in this area is probably needed; the black objects are probably simply absorbing most of the laser energy and 
reflecting very little. It was noticed that the laser would also return signal strength of zero when targeting some dark, flat panels held at certain angles to the beam.

\section{CONCLUSION}

A series of tests have been designed to assess the performance of individual sensors relative to the requirements of pre-crash sensing. A selection of test results for an Ultrasonic and LIDAR sensors have also been presented. The data to-date indicate that these sensors are complementary in performance (at significantly different ranges), but are insufficient to form a complete sensor system. Since the LIDAR sensor cannot detect all types of objects, it does not provide enough data at the long range. In addition, testing has not yet identified a viable method to discriminate between object types - a long-term goal of any precrash sensing system.

These test results will be used to direct the design of a pre-crash sensing system integrating the responses of multiple sensors. In particular, to address the issues identified with the two tested sensors, a Radar sensor is currently being tested. The resulting system should take advantage of the strengths of each and overlap the weaknesses of others. Combined with an intelligent algorithm the system should provide real-time information to an automobile computer to enable improvement in current safety technology and facilitate development and deployment of the next generation of safety technologies. The end results is that vehicle crash survivability will be increased, saving lives.

\section{ACKNOWLEDGMENTS}

This work was funded by a grant from the California Central Coast Research Partnership.

\section{REFERENCES}

1. Carlin, J., Birdsong, C., Schuster, P., Thompson, W. and Kawano, D., (2005), "Evaluation of Cost Effective Sensor Combinations for a Vehicle PreCrash Detection System," SAE Commercial Vehicle Engineering Congress \& Exhibition, Chicago, November 1-3, 2005.

2. Jones RC (2002), "Technologies for Static Airbag Suppression Systems", 6th International Symposium on Sophisticated Car Occupant Safety Systems, Karlsruhe, Germany, December 2-4, 2002.

3. Knoll P, Schaefer BJ, Guettler H, Bunse $M$, Kallenbach R (2004), "Predictive Safety Systems Steps Towards Collision Mitigation." SAE 2004 World Congress, Detroit, MI, USA.

4. Lyons CT \& Taskin I (2000), "A low-cost MMIC based radar sensor for frontal, side or rear automotive anticipatory pre-crash sensing applications," IEEE Intelligent Vehicles Symposium, Dearborn, MI, USA.

5. NHTSA (2005), "Traffic Safety Facts 2003." DOT HS 809 775, www.nhtsa.dot.gov.

6. Spies HD (2002), "What is Achievable Today and in the Near Future? - Overview on Technologies: Radar, Video, IR." 6th International Symposium on Sophisticated Car Occupant Safety Systems, Karlsruhe, Germany, December 2-4, 2002. 